\section{Efectos de un programa de entrenamiento multicomponente en la marcha funcional en pacientes con Parkinson}

\author{
MARCELA GAZMURI CANCINO ${ }^{1, a}$, ÉRICA REGALADO VÁSQUEZ ${ }^{1, a}$, \\ GUSTAVO PAVEZ-ADASME²,b ${ }^{2,}$ CLAUDIO HERNÁNDEZ-MOSQUEIRA ${ }^{2,3,4, c}$
}

\section{Multicomponent physical training in patients with Parkinson disease}

Background: Multicomponent physical training in patients with Parkinson disease may improve their functional independence, especially in terms of gait speed and coordination. Aim: To assess the effects of an eight weeks multicomponent physical training program in patients with Parkinson disease. Material and Methods: Fourteen patients with Parkinson disease participated in a physical training program that lasted eight weeks. Three sessions lasting 60 minutes per week were carried out. Patients were assessed using the six minutes walk, timed up and go and the unipodal stance test. Results: After the training period, significant improvements in the six minute walk test and timed up and go were observed. No significant changes were observed in the unipodal stance test. Conclusions: The eight weeks training program improved gait speed and functional status in these patients with Parkinson disease.

(Rev Med Chile 2019; 147: 465-469)

Key words: Exercise; Gait; Parkinson Disease; Physical Conditioning.
'Universidad Mayor. Santiago,

Chile.

${ }^{2}$ Grupo de Investigación AFSYE,

Pedagogía en Educación Física,

Universidad Adventista de Chile.

Chillán, Chile.

${ }^{3}$ Departamento Ciencias de la Actividad Física, Universidad de Los Lagos. Puerto Montt, Chile. ${ }^{4}$ Laboratorio de Biociencias del Movimiento Humano (LABIMH),

Escuela de Educación Física, Universidad Federal de Río de Janeiro, Brasil.

aKinesiólogo, Magíster en Fisiología Clínica del Ejercicio. bProfesor de Educación Física, Magíster en Salud y Bienestar Humano.

'Profesor de Educación Física, Doctor en Ciencias de la Motricidad Humana.

Trabajo no recibió financiamiento. Los autores declaran no tener conflictos de interés.

Recibido el 27 abril de 2018, aceptado el 1 de febrero de 2019

Correspondencia a: Gustavo Pavez-Adasme Grupo de Investigación AFSYE, Pedagogía en Educación Física, Universidad Adventista de Chile. gustavopavez@unach.cl
L a Enfermedad de Parkinson (EP) se define como una enfermedad crónica, neurodegenerativa y progresiva, caracterizada por la pérdida de las vías dopaminérgicas nigroestriales ${ }^{1}$, que afecta de sobremanera la calidad de vida de quienes la padecen llegando a perder inclusive su propia autonomía e independencia. En Chile, la mortalidad por EP en la población mayor a 65 años es alrededor de $1 \%-2 \%$ y a medida que se acerca a los 85 años aumenta entre $3 \%-5 \%{ }^{2}$. Aunque actualmente la EP no cuenta con tratamiento curativo, los síntomas se tratan con fármacos que reponen la dopamina deficitaria, los que son insuficientes para frenar y enlentecer el curso degenerativo de la $\mathrm{EP}$, afectando así la velocidad de la marcha, tan im- portante para la funcionalidad e independencia ${ }^{3} . \mathrm{La}$ aplicación de un programa de entrenamiento en EP podría retrasar la discapacidad en la enfermedad ${ }^{4}$, por ello el objetivo de este estudio fue determinar los efectos de un programa de entrenamiento multicomponente de 8 semanas en la marcha funcional de los enfermos con Parkinson de la agrupación de Parkinson de Chillán.

\section{Material y Método}

Estudio de tipo pre-experimental, con diseño pre/post test con un solo grupo de muestra intencionada, aplicado a EP durante 8 semanas. 


\section{Sujetos}

La muestra incluyó a 14 sujetos adultos y adultos mayores pertenecientes a la agrupación de Parkinson de Chillán (9 varones y 5 mujeres). Todos los participantes del estudio cumplieron los criterios de inclusión: paciente diagnosticado de EP en estadios 1-3 (Escala de Hoehn y Yahr) ${ }^{5}$, no presentar demencia, capacidad de deambulación de manera independiente, no alteración de los reflejos posturales, firmar el consentimiento informado, no presentar ninguna contraindicación médica al respecto y aceptación de las normas del estudio (asistencia regular y participación activa). Los criterios de exclusión fueron los siguientes: enfermedad neurológica que suponga la no comprensión de órdenes verbales u otra que influya en el resultado obtenido, lesiones articulares y/o musculares de miembros inferiores que afectasen a la marcha autónoma.

\section{Consideraciones éticas}

Los sujetos de estudio fueron invitados a participar de este proyecto una vez aprobados por el comité de Bioética de la Universidad Adventista de Chile Dictamen y firmado el consentimiento informado respectivo para la participación de este estudio de acuerdo con la Declaración de Helsinki, antes de iniciar las evaluaciones iniciales.

\section{Procedimientos}

La intervención del estudio tuvo una duración de 8 semanas, donde se entrenó a los sujetos con una combinación de ejercicios multicomponentes, 3 veces por semana, con una duración $60 \mathrm{~min}$ cada sesión de entrenamiento. Previo a la intervención se aplicaron una serie de instrumentos de evaluación, para poder observar los cambios del plan de entrenamiento. Test de marcha de los 6 minutos, Timed Up and Go, Estación Unipodal y estadio Hoehn y Yahr. El diseño del plan de entrenamiento multicomponente que se aplicó a los sujetos de estudio se distribuyó de la siguiente manera (Figura 1).

\section{Materiales e instrumentos de valoración}

Para la valoración y medición de los sujetos de estudio previo a la realización del programa de entrenamiento multicomponente de 8 semanas se utilizaron los siguientes instrumentos de valoración:

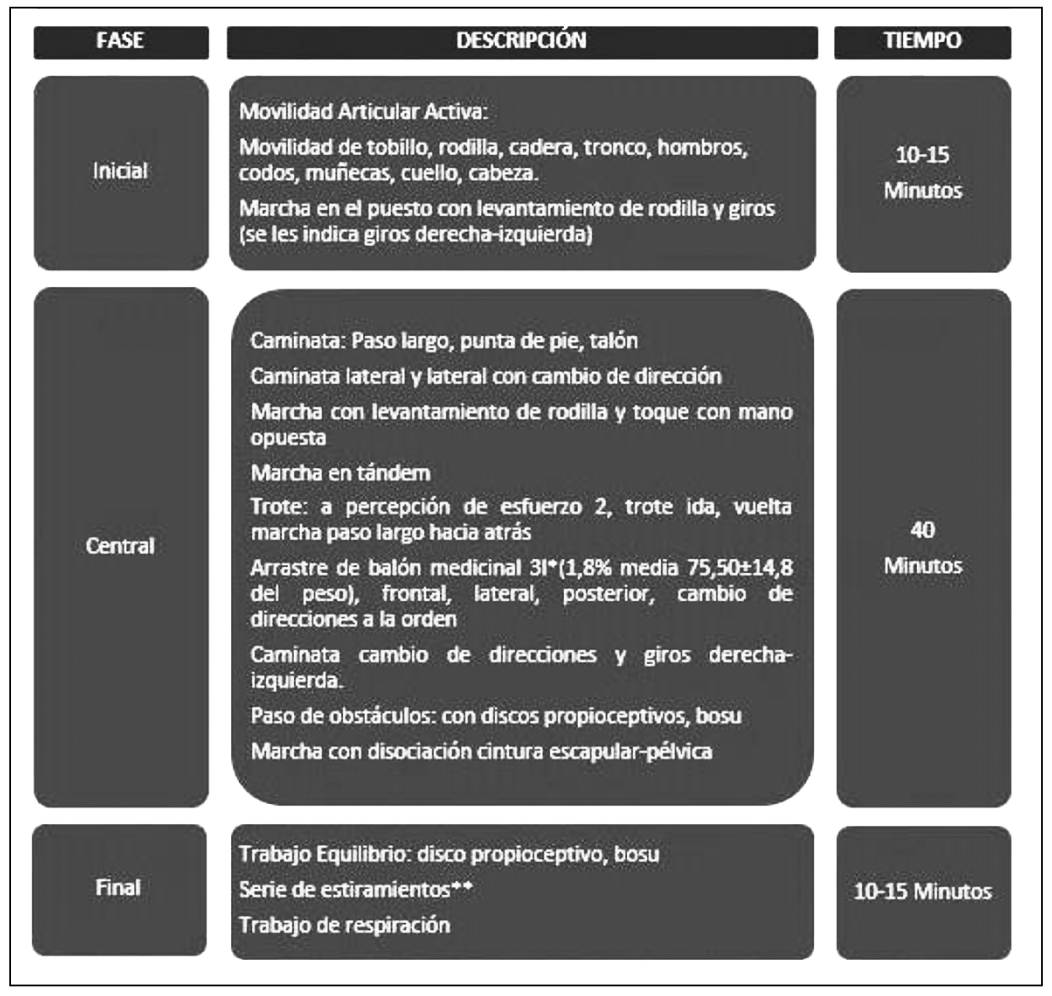

Figura 1. Distribución del entrenamiento multicomponente. *Desplazamientos con balones medicinales blandos 3 libras $(1,8 \%$ del peso corporal, media 75,50 \pm 14,8). **Estiramientos: Abductores, aductores, flexión de tronco en sedestación, cuádriceps, gastrocnemios y musculatura lumbar. 
- Test de marcha de los 6 minutos (TM6') ${ }^{6}$.

- Escala del esfuerzo percibidode Borg ${ }^{7}$.

- Prueba de Estación Unipodal (EUP) y Timed Up and Go (TUG) ${ }^{8}$.

\section{Método de análisis de datos}

Se calcularon las medias y desviaciones típicas para definir las características de la muestra, la normalidad se realizó a través de la prueba de Shapiro- Wilk. Con el fin de identificar los efectos del programa multicomponente sobre las variables, para los datos que no se distribuyeron normalmente, se aplicó la prueba de rangos con signo de Wilcoxon y para los que presentaron normalidad se utilizó t de Student para pruebas relacionadas,mientras que el tamaño del efecto (TE) se calculó utilizando la prueba d de Cohen. Todos los análisis consideraron un valor $\mathrm{p}<0,05$ estadísticamente significativo y se realizaron con el programa estadístico IBM ${ }^{\circledR}$ SPSS $^{\circledR}$ Statistics v. 21 .

\section{Resultados}

En la Tabla 1, se presentan las características de la población estudiada y la distribución porcentual en la clasificación de estadios de Hoehn y Yahr, donde un mayor porcentaje de pacientes se encuentran en el estadio I.

Aplicada la prueba t de Student en el TM6', se observaron cambios significativos en la distancia recorrida entre el pre test y post test $(\mathrm{p}=0,002)$ y un TE bajo $=0,25$. En relación al TUG también se observan cambios significativos $(p<0,000)$ y un TE moderado $=0,60$. En cuanto a los tiempos obtenidos en la prueba de EUP, estos no presentan cambios significativos (Tabla 2).

Tabla 1. Características de la población estudiada y su clasificación por estadios

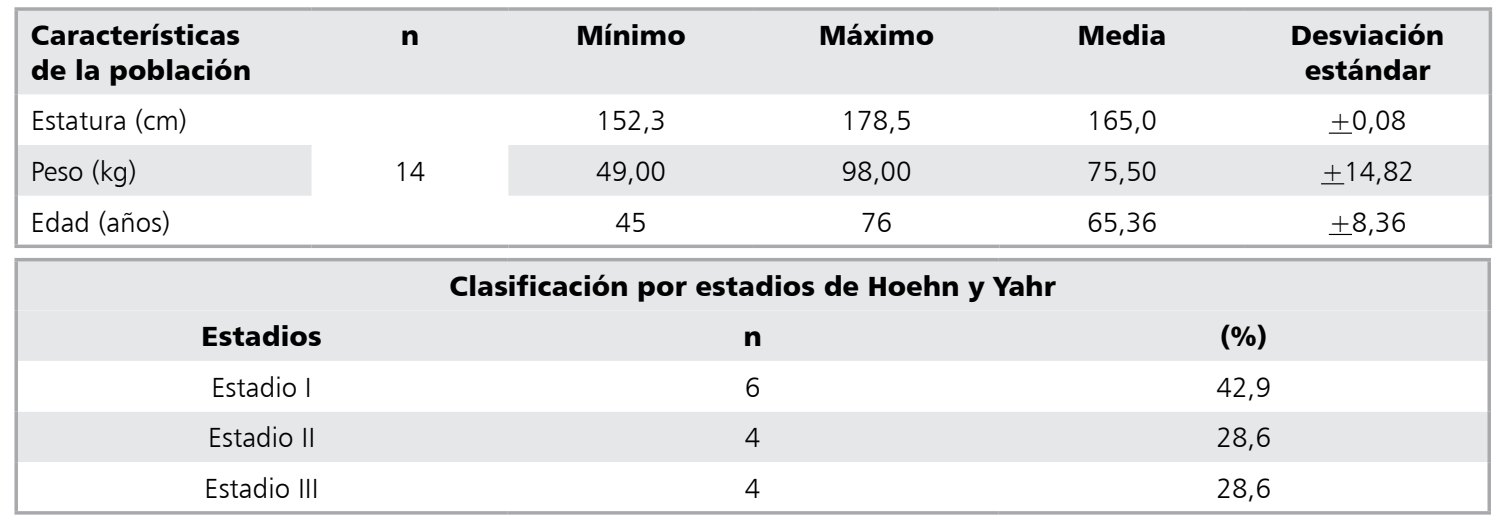

Tabla 2. Medias obtenidas en Test de marcha de los 6 minutos, Timed Up and Go, estación unipodal de pie derecho e izquierdo al inicio y final de la intervención

\begin{tabular}{|c|c|c|c|c|c|c|}
\hline & $\mathbf{n}$ & Test & Media & DS & Valor p & TE \\
\hline \multirow[t]{2}{*}{ TM6'(mts) } & \multirow{2}{*}{14} & Pre & 476,86 & 89,93 & \multirow[t]{2}{*}{$0,002^{*}$} & \multirow[t]{2}{*}{0,25} \\
\hline & & Post & 521,86 & 87,01 & & \\
\hline \multirow[t]{2}{*}{ Time Up and Go (seg) } & \multirow{2}{*}{14} & Pre & 7,29 & 1,70 & \multirow[t]{2}{*}{$0,000^{*}$} & \multirow[t]{2}{*}{0,60} \\
\hline & & Post & 5,31 & 0,80 & & \\
\hline \multirow[t]{2}{*}{ Estación Unipodal Pie Derecho (seg) } & \multirow{2}{*}{14} & Pre & 7,92 & 2,88 & \multirow{2}{*}{$0,45^{a}$} & \multirow[t]{2}{*}{0,13} \\
\hline & & Post & 8,57 & 1,91 & & \\
\hline \multirow[t]{2}{*}{ Estación Unipodal Pie Izquierdo (seg) } & \multirow{2}{*}{14} & Pre & 8,53 & 2,75 & \multirow[t]{2}{*}{$0,87^{a}$} & \multirow[t]{2}{*}{0,03} \\
\hline & & Post & 8,38 & 2,01 & & \\
\hline
\end{tabular}

*Nivel de significancia utilizando la prueba t Student $(p<0,05)$. $a=$ Nivel de significancia utilizando la prueba de Signos de Wilcoxon $(p<0,05)$. TE $=$ Tamaño del efecto. 

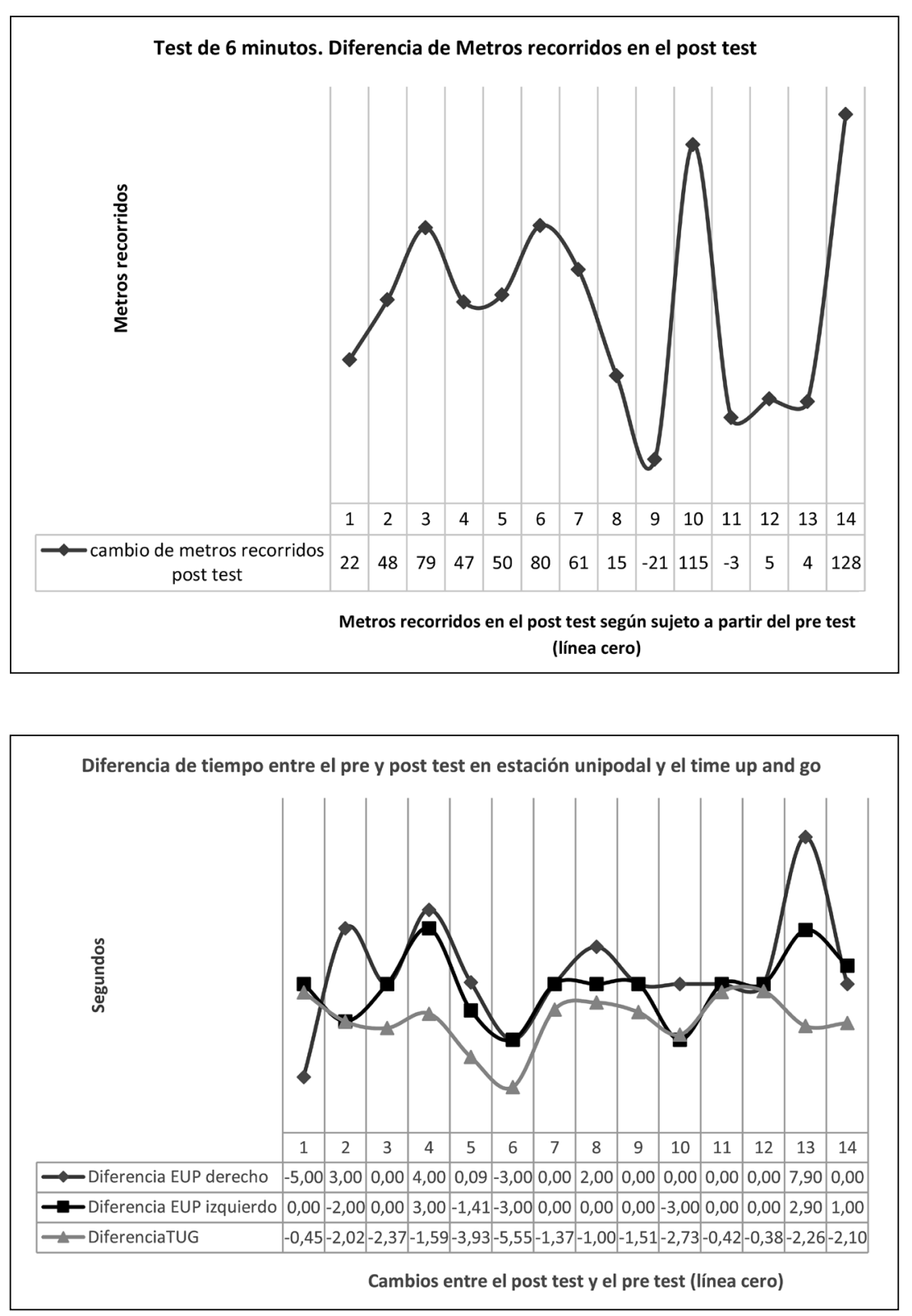

Figura 2. Cambios observados en los pacientes tras la aplicación de pre y post test de Marcha de los 6 min.
Figura 3. Cambios observados en los sujetos tras la aplicación de pre y post test en Estación Unipodal (EUP) pie derecho e izquierdo y Timed Up and Go (TUG).
En las Figuras 2 y 3 se presentan los cambios observados por cada uno de los participantes en las pruebas realizadas, se aprecia que en el test de marcha $85,71 \%$ de los pacientes mejoraron su desempeño en los metros recorridos en el test, a diferencia de las pruebas de equilibrio estático y dinámico donde observamos que en las pruebas de estación unipodal pie derecho e izquierdo menos de $50 \%$ de los evaluados mejoraron en estas pruebas ( $42,85 \%$ y $21,42 \%$ respectivamente). Finalmente, en la prueba de equilibrio dinámico Timed up and go el $100 \%$ de los pacientes mejoraron su desempeño.

\section{Discusión}

El entrenamiento multicomponente es el tipo de ejercicio físico más beneficioso no solo para 
el anciano frágil, si no que su aplicación genera beneficios a una población con $\mathrm{EP}^{9}$. En nuestro estudio se observan diferencias significativas $(\mathrm{p}=0,002)$ para aquellos pacientes sometidos al plan de entrenamiento multicomponente en el TM6, sin embargo, se observa un bajo tamaño del efecto $(0,25)$. Similares resultados son reportados por Rafferty et $\mathrm{a}^{10}$, quienes observaron mejoras a largo plazo en la velocidad de marcha rápida en personas con EP leve y moderada, con la implementación de un programa de entrenamiento progresivo de resistencia. Al tener una marcha más lenta y sin estimulación, este tipo de paciente, pueden llegar a presentar avances de su enfermedad, sufriendo con ello riesgos de fragilidad, como lo son las caídas y perdidas de equilibrio, generando más momentos de Freezing o congelamiento de la marcha ${ }^{11}$, estudios que relacionan el riesgo de caída y la $\mathrm{EP}$, mencionan que entre $70 \%$ y $87 \%$ de los EP, caen en algún momento ${ }^{12}$. Según Sai et al. ${ }^{13}$, el TUG es el mejor test de equilibrio para predecir caídas recurrentes en este tipo de pacientes. En nuestros pacientes se observaron diferencias significativas $(p=0,000)$, tras la aplicación del plan de entrenamiento multicomponente en el TUG. Similares son los resultados reportados por Carpinella et $\mathrm{al}^{14}$, quienes reportan diferencias significativas $(\mathrm{p}=0,047)$, en desempeños de equilibrio del grupo experimental sometido a un entrenamiento de equilibrio y marcha con 20 sesiones de duración. Diferente situación ocurrió en la prueba de EUP, donde nuestro estudio no reporto diferencias significativas para el pie derecho $(\mathrm{p}=0,45)$ y para el pie izquierdo $(p=0,87)$. Sin embargo, es importante la mantención de una buena estabilidad, así lo menciona Mancilla et $\mathrm{al}^{8}$, ya que a mayor edad del adulto mayor, el tiempo de EUP es menor, por lo cual tiene mayor riesgo de caída en este tipo de pacientes.

Con base en los resultados de este estudio se puede concluir que la aplicación de un programa de entrenamiento multicomponente de 8 semanas parece ser un tratamiento beneficioso para los pacientes diagnosticados con EP leve y moderado, ya que genera cambios significativos en la funcionalidad de marcha de este tipo de pacientes.

Agradecimientos: A la Agrupación del Parkinson de Chillán, pacientes, familiares y colaboradores que aportaron un gran apoyo al estudio.

\section{Referencias}

1. Fahn S. Description of Parkinson's disease as a clinical syndrome. Ann N Y Acad Sci 2003; 991: 1-14.

2. Chaná P, Jiménez M, Díaz V, Juri C. Mortalidad por enfermedad de Parkinson en Chile. Rev Med Chile 2013; 141(3): 327-31.

3. Shulman LM, Katzel LI, Ivey FM, Sorkin JD, Favors $\mathrm{K}$, Anderson KE et al. Randomized Clinical Trial of 3 Types of Physical Exercise for Patients With Parkinson Disease. JAMA Neurol 2013; 70 (2): 183-90.

4. King LA, Horak FB. Delaying Mobility Disability in People With Parkinson Disease Using a Sensorimotor Agility Exercise Program. Phys Ther 2009; 89 (4): 384-93.

5. Hoehn M, Yahr M. Parkinsonism onset, progression, and mortality. Neurology 1967; 17 (5): 427-42.

6. Beroíza WT, Cartagena SC, Caviedes SI, Céspedes G J, Gutiérrez-Navas M, Oyarzún GM, et al. Prueba de caminata de seis minutos. Rev Chil Enferm Respir 2009; 25 (1): 15-24.

7. Borg GA. Psychophysical bases of perceived exertion. Med Sci Sports Exerc 1982; 14 (5): 377-81.

8. Mancilla E, Valenzuela J, Escobar M. Rendimiento en las pruebas "Timed Up and Go" y "Estación Unipodal" en adultos mayores chilenos entre 60 y 89 años. Rev Med Chile 2015; 143 (1): 39-46.

9. Izquierdo M, Cadore E, Casas-Herrero A. Ejercicio físico en el anciano frágil: una manera eficaz de prevenir la dependencia. Rev Kronos 2014; 13 (1): 1-14.

10. Rafferty MR, Prodoehl J, Robichaud JA, David FJ, Poon C, Goelz LC, et al. Effects of 2 Years of Exercise on Gait Impairment in People With Parkinson Disease: The PRET-PD Randomized Trial. J Neurol Phys Ther 2017; 41 (1): 21-30.

11. Amboni M, Barone P, Picillo M, Cozzolino A, Longo K, Erro R, et al. A two-year follow-up study of executive dysfunctions in Parkinsonian patients with freezing of gait at on-state. Mov Disord 2010; 25 (6): 800-2.

12. Hely MA, Reid WGJ, Adena MA, Halliday GM, Morris JGL. The Sydney multicenter study of Parkinson's disease: The inevitability of dementia at 20 years. Mov Disord 2008; 23: 837-44.

13. Sai A, Gallagher JC, Smith LM, Logsdon S. Fall predictors in the community dwelling elderly: a cross sectional and prospective cohort study. J Musculoskelet Neuronal Interact 2010; 10 (2): 142-50.

14. Carpinella I, Cattaneo D, Bonora G, Bowman T, Martina L, Montesano A, et al. Wearable Sensor-Based Biofeedback Training for Balance and Gait in Parkinson Disease: A Pilot Randomized Controlled Trial. Arch Phys Med Rehabil 2017; 98 (4): 622-30.e3. 